\title{
Improvement of an Extended Kalman Filter Power Line Interference Suppressor for ECG Signals
}

\author{
LD Avendaño-Valencia ${ }^{1}$, LE Avendaño ${ }^{2}$, JM Ferrero $^{3}$, G Castellanos-Domínguez ${ }^{1}$ \\ ${ }^{1}$ Universidad Nacional de Colombia, Manizales, Colombia \\ ${ }^{2}$ Universidad Tecnológica de Pereira, Pereira, Colombia \\ ${ }^{3}$ Universidad Politécnica de Valencia, Valencia, España
}

\begin{abstract}
The powerline interference reduction in ECG records is a challenging problem which is still open for research. The powerline signal, measured directly from the transmission line may have amplitude, phase and frequency variations. These reasons make the classical filtering methods suboptimal in the powerline interference reduction. We propose a tracking method based on Kalman filtering which uses an state space model for the noisy signal and allows adequate discrimination between the ECG signal and the perturbation, even during non-stationarities. The parameters of this algorithm are optimized via genetic algorithms, obtaining a set of values that give it a mean correlation index on the QT database over 0,99 .
\end{abstract}

\section{Introduction}

The sinusoidal interference reduction on a time series such as electrocardiogram (ECG) is a serious restriction which still is an open research topic. The powerline interference is particulary annoying in medical systems, because can totally mask the signal of interest rendering it useless for heart condition diagnosis. Most of the works are founded on the fact that the sinusoidal signal is an stationary process [1]. However, the powerline signal, measured directly from the transmission line, has variations in amplitude, frequency and phase, along with the presence of harmonics with time-varying characteristics as well [2].

In this sense, the filtering of these interferences also requires tracking of their parameter variations. Particularly, an approach to remove the powerline interference consists on the estimation of amplitude and phase in an isoelectric segment, followed by subtraction of the estimated sinusoid within the entire heartbeat [3]. The performance of this approach, known as estimation-subtraction $(E-S)$ method, deteriorates when parts of the $P$ or $Q$ wave are included in the segment. The sinusoid is subtracted not only from the interval in which it is estimated, but also for the remaining parts of the heartbeat. Such beat-to-beat oriented procedure implies, however, that sudden shifts in amplitude may occur in the output signal at the boundaries of successive beats [3]. A different approach consists on a non-linear least minimum squares algorithm which estimates the amplitude, frequency and phase of a non-stationary sinusoid (non-linear adaptive estimator of non-stationary sinusoids NAENS) [4], and that has been adapted to reduce the power line interference on ECG. It has been shown that it is a simple and fast estimation procedure, but with modest performance for cases of low signal to noise ratios.

The principal shortcomings of these algorithms, based on least minimum squares, come from their lack of robustness in the estimation procedure and insufficient discrimination between the ECG signal and the powerline interference. These factors motivate the application of more effective estimation algorithms such as the Kalman filter, which has the ability to separate the signal components from noise, and overcome the difficulties of non-stationarity [5]. This can be carried out using a proper model to describe a sinusoidal signal, which is applied to the Kalman filter.

In this paper, we propose a tracking method, based on Kalman filtering, which uses an state space model for the noisy signal, and allows adequate discrimination between the ECG signal and the perturbation even during nonstationarities. Specifically, a model, which was proposed to track the amplitude, frequency and phase of a quasistationary sinusoid $[6,7]$, is used to reduce the powerline interference by adding another state encoding the ECG dynamics. Kalman filtering parameters are tuned by means of a simple genetic algorithm ( $S G A$ ) that explores the parameter space related with the correlation index of the filtered records of the $Q T$ database. As a result, it is shown that the efficiency of the proposed filter, with optimized parameters, overcomes the performance of the estimationsubtraction and NAENS algorithms for stationary interference and assuming both amplitude and frequency changes, as well. Moreover, performance of the filter turns to be high for a large span of parameters. 


\section{Methods}

\subsection{Model for signal and interference}

The following nonlinear signal model describes the evolution of noisy quadrature data with a slowly time-varying frequency $[6,7,8]$ :

$$
\begin{aligned}
x_{1}[n+1] & =x_{1}[n] \cos \left(x_{3}[n]\right)-x_{2}[n] \sin \left(x_{3}[n]\right)+w_{a}[n] \\
x_{2}[n+1] & =x_{1}[n] \sin \left(x_{3}[n]\right)+x_{2}[n] \cos \left(x_{3}[n]\right)+w_{a}[n] \\
x_{3}[n+1] & =(1-\epsilon) x_{3}[n]+w_{f}[n] \\
x_{4}[n+1] & =x_{4}[n]+w_{e c g}[n] \\
y[n] & =x_{1}[n]+x_{4}[n]+v[n], n \in \mathbb{Z},
\end{aligned}
$$

where $x_{3}$ is a unknown time-varying frequency, $x_{1}$ and $x_{2}$ are in-phase and quadrature signals. The ECG signal is encoded in the state $x_{4}$ as a first order autoregressive process, whose spectral density has high resemblance with the spectral density of the ECG signal. Besides, $\mathbf{w}[n]=\left[w_{a}[n] w_{a}[n] w_{f}[n] w_{e c g}[n]\right]^{\top}$ is white Gaussian noise with zero mean and covariance matrix, defined as:

$$
\begin{aligned}
E\left\{\mathbf{w}[n] \mathbf{w}^{\top}[m]\right\} & =\left\{\begin{array}{llll}
\mathbf{Q}, & n=m ; \\
\mathbf{0}, & n \neq m .
\end{array}\right. \\
\mathbf{Q} & =\left[\begin{array}{cccc}
\sigma_{a}^{2} & 0 & 0 & 0 \\
0 & \sigma_{a}^{2} & 0 & 0 \\
0 & 0 & \sigma_{f}^{2} & 0 \\
0 & 0 & 0 & \sigma_{e c g}^{2}
\end{array}\right]
\end{aligned}
$$

and $v[n]$ is white Gaussian noise with zero mean and variance, defined as:

$$
E\left\{v[n] v^{\top}[m]\right\}= \begin{cases}r, & n=m ; \\ 0, & n \neq m .\end{cases}
$$

The parameter $\epsilon \geq 0$, typically $\epsilon \ll 1$, has the goal of enforcing stability into Eq. (1) and determines the rate of time variation of $x_{3}$. It should be chosen so that the frequency varies slowly enough to make the signal appear periodic over several cycles.

The procedure to extract the sinusoid from ECG involves the estimation of the clean ECG signal and the interfering sinusoid from the original noisy signal with the extended Kalman filter ${ }^{1}$ using the state space model described in Eq. (1). Then, the clean ECG signal can be extracted from the fourth state $x_{4}[n]$.

The Kalman filter has three parameters related to the convergence and noise rejection capabilities of the estimation. They are process noise covariance $\mathbf{Q}$, measurement noise variance $r$ and initial error covariance $\mathbf{P}$. It is shown, that the elements of $\mathbf{Q}$ and $r$ are related by the quantity $\lambda=r / \sigma^{2}$ [7]. In this way, when $\lambda$ increases the error variance diminishes, however, its tracking ability deteriorates;

\footnotetext{
${ }^{1}$ For a complete description of the extended Kalman filter, see [5]
}

in opposition, when $\lambda$ decreases, the tracking process becomes more robust for state variations, but the error variance grows.

The process noise covariance is composed by three constants $\sigma_{a}^{2}, \sigma_{f}^{2}$ and $\sigma_{e c g}^{2}$. The first one is the variance of the noise of the amplitude process, the second term is the variance of the noise of the frequency process while the third term is the variance of the useful ECG signal. Thus, $\lambda_{a}=r / \sigma_{a}^{2}, \lambda_{f}=r / \sigma_{f}^{2}$ and $\lambda_{e c g}=r / \sigma_{e c g}^{2}$, which are selected depending on the expected characteristics of the filter.

On the other hand, the components of $\mathbf{P}$ adjust the convergence rate of the filter. Its values should be large for fast convergence, but may lead to divergence if the initial estimated state is close to the real state; by contrast, setting small values will give more stability with long convergence time. Tuning of this parameters is generally carried out empirically. In this case employment of a GA is proposed in order to optimize the performance of the filter in some sense, as described in the following section.

\subsection{Parameter tuning by genetic algorithms}

The genetic algorithm used, the Simple Genetic Algorithm ( $S G A)$, is a method to move through chromosomes populations using a sort of natural selection and the crossover and mutation operators, inspired by genetics. Each possible combination of Kalman filter parameters can be codified in a finite length chain, known as chromosome. In [9] is shown that the best form of chromosome is a binary chain, as follows:

$$
\mathbf{c}=\left[\mathcal{C}\left\{\lambda_{\infty}\right\}, \ldots, \mathcal{C}\left\{\lambda_{\mathcal{M}}\right\}\right]
$$

where $\mathbf{c}$ is the resulting chromosome and $\mathcal{C}$ is an operator of conversion to binary system.

The initial population $P_{0}=\left\{c_{1}, \ldots, c_{r}\right\}$ of $r$ chromosomes is generated randomly. The parameter $r$, or initial population length, is a control parameter to be tuned in order to get a proper performance of the $S G A$. Then the selection of best chromosomes is done assigning to each individual a fitness value by means of a ranking function [10]. Particularly, exponential ranking is considered, which, after ordering the individuals from 1 to $r$ (where 1 is the worst qualified and $r$ is the best qualified), assigns a quality value according to $f\left(\mathbf{c}_{\mathbf{i}}\right)=\mathbf{s}^{\mathbf{r}-\mathbf{i}}$ where $s \sim 1$. Based on this quality value, the fittest individuals are chosen by means of stochastic sampling [11].

Then, the recombination process is accomplished for random paired chromosomes having a $\chi$ probability. The $S G A$ uses single point recombination, which consists of two chains for a single random sampled point to exchange chain segments. After recombination, a mutation operator is applied for a $\mu$ probability value, which is done by bit 
negation. The probability amounts, $\chi$ and $\mu$, are also control parameters. The selection, recombination and mutation stages are sequentially repeated until $95 \%$ of the population have converged to a solution chromosome [12].

\section{Experimental setup and results}

Database: The $Q T$ Database includes ECG recordings which were chosen to represent a wide variety of $Q R S$ and $S T-T$ morphologies, in order to challenge ECG signal processing algorithms with real-world variability. The records were chosen primarily among existing ECG databases which are a wide source of varied and well characterized data, including reference annotations marking the location of waveform boundaries. The $Q T$ Database contains a total of 105 fifteen-minute excerpts of two channel ECGs, selected to avoid significant baseline wander or another artifacts. All records were sampled at $250 \mathrm{~Hz}$. Those which were not originally sampled at that rate were converted using a sub-sampling software.

Simulation of powerline interference: The $Q T$ database records are artificially contaminated with the following power line interference model:

$$
y[k]=A[k] \sin \left(\frac{2 \pi f[k]}{f_{s}} k+\phi_{0}\right)
$$

where $A[k]=A_{0}+\xi_{A}[k]$ and $f[k]=f_{0}+\xi_{f}[k], A_{0}$ and $f_{0}$ are amplitude and base frequency, and $\xi_{A}[k]$ and $\xi_{s}[k]$ correspond to white Gaussian noise, which represent the variations about these base quantities. The value for $A_{0}$ is set according to the signal to noise ratio, while $f_{0}$ is the powerline frequency.

Test description: The proposed algorithm is tested filtering the contaminated records of the $Q T$ database. The filter's performance is measured with the correlation index, defined as [13]:

$$
\rho_{x y}=\frac{C_{x y}}{\sqrt{C_{x x} C_{y y}}}
$$

where $C_{x y}$, is the cross-covariance of $x$ and $y$, and $C_{x x}$ is the covariance of $x$. To know the filter's response for different ECG morphologies, this index is measured on the entire $Q T$ database. For sake of benchmarking, we also do this test with E-S [3] and NAENS [4].

\subsection{Parameter settings}

The parameter space is obtained as the mean and variance of the correlation index of the original and filtered records of the $Q T$ database for different parameter combinations. The optimized parameters are $\lambda_{a}$ and $r$. In Fig.1 the parameter space for a signal to noise ratio of $3 d B$ is shown. The genetic algorithm uses the information of this surface to obtain a search space. The final optimized parameters are $\lambda_{a}=10^{-3}$ and $r=10^{-3}$ with a correlation index $\rho=0,9975$, for $\epsilon=0, \lambda_{f}=5 \times 10^{-5}$, $\lambda_{e c g}=0,1 \lambda_{a}$.

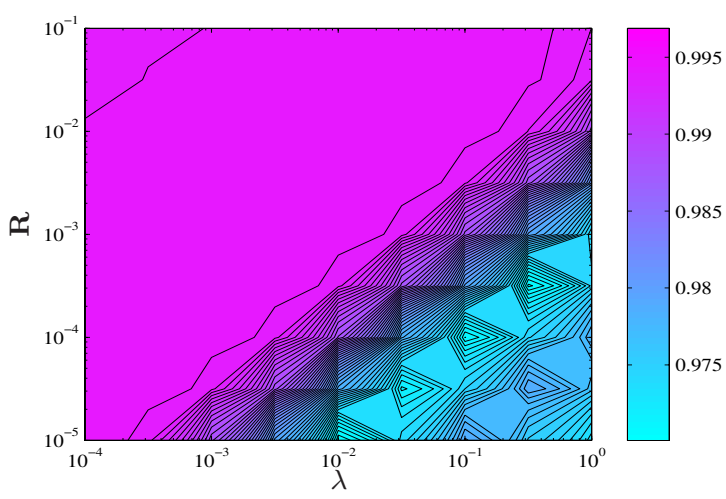

Figure 1. Mean correlation for parameter space.

\subsection{Filtering results}

Figures 2(a)-(d) show the correlation index of the records of the complete $Q T$ database by means of boxplots, which show the dispersion around the median correlation.

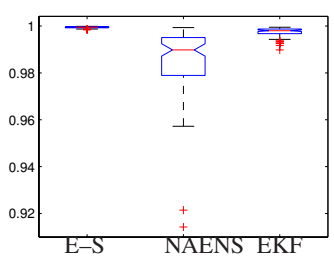

(a)Stationary interference.

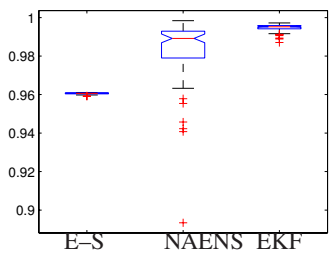

(c)Time-varying frequency.

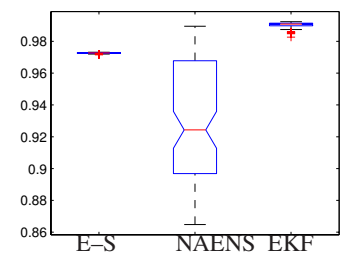

(b)Time-varying amplitude.

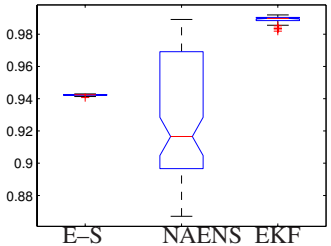

(d)Joint variations.
Figure 2. Correlation index for the $Q T$ database.

Figures 3(a), (b) and (c) show the filtering results of NAENS, E-S and the proposed algorithm. Figure 3(a) shows the filtering results on a normal segment of ECG signal with $S N R=3 d B$ stationary powerline interference. Figure 3(b) shows the behavior of the filters during an amplitude change. In this simulation, initially there is no interference and at $t=3,8 \mathrm{~s}$ the interference's amplitude gradually increases. Figure 3(c) shows the behavior of the filters during a frequency change. Here, the frequency of 


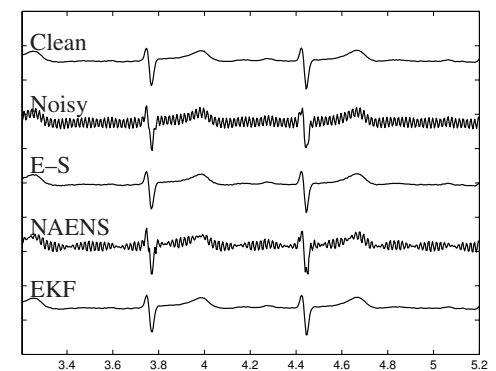

(a) Steady state.

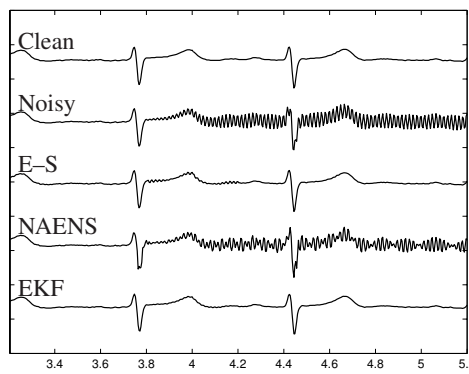

(b)Amplitude transient.

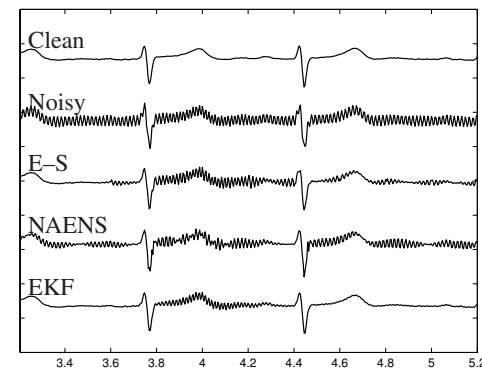

(c)Frequency transient.

Figure 3. Filter response for different situations.

the powerline interference changes from $60 \mathrm{~Hz}$ to $61 \mathrm{~Hz}$ at $t=3,8 \mathrm{~s}$.

\section{Discussion and conclusions}

The proposed non-stationarity tracking approach for the powerline interference by means of Kalman filtering allows improved discrimination between the ECG signal and the perturbation. This method properly reduces this interference during its stationary segments and keeps its high performance during amplitude, frequency and joint variations. One of the main shortcomings of the Kalman filter methods, the parameter setting, was proposed to be solved with the implementation of a SGA, obtaining a set of parameters, being optimal in the correlation index sense. The search surface shows that the EKF powerline interference suppressor has high performance in a large span of the parameter space. The database tests show that this set of parameters is optimal for the enclosed ECG morphologies.

\section{Acknowledgements}

This work was partially supported by the project Técnicas de computación de alto rendimiento en la interpretación automatizada de imágenes médicas y bioseñales, financed by DIMA and VRI of Universidad Nacional de Colombia, code 20201004224.

\section{References}

[1] Pei S, Tseng C. Elimination of AC interference in electrocardiogram using IIR notch filter with transient suppression. IEEE Transactions on Biomedical Engineering November 1995;42:1128-1132.

[2] Sedlàcek M, Blaska J. Low uncertainty power-line frequency estimation for distorted and noisy harmonic signals. Elsevier Measurement 2004;

[3] Sörnmo L, Laguna P. Bioelectrical Signal Processing in Cardiac and Neurological Applications. Elsevier Academic Press, 2005.

[4] Ziarani A, Konrad A. A nonlinear adaptive method of elim- ination of power line interference in ECG signals. IEEE Transactions on Biomedical Engineering 2002;

[5] Haykin S. Kalman Filtering and Neural Networks. Adaptive and learning systems for signal processing, communications and control, first edition. Wiley Interscience, 2001.

[6] Scala BL, Bitmead RR. Design of an extended Kalman filter frequency tracker. IEEE Transactions on Signal Processing Vol 44 No 3 1996;

[7] Bittanti S, Savaresi SM. On the parameterization and design of an extended Kalman filter frequency tracker. IEEE Transactions on Automatic Control Vol 45 No 9 2000;.

[8] Avendaño E, Avendaño D, Castellanos C, Villegas E. Reduction of power line interference in ECG signals using Kalman filtering and delta operator. 23rd ISPE International Conference on CADCAM Robotics and Factories of the Future 2007;2:813-817.

[9] Holland JH. Adaptation in Natural and Artifical Systems. Ann Arbor, MI: University of Michigan Press, 1975.

[10] Zhang BT, Kim JJ. Comparison of selection methods for evolutionary optimization, 2000.

[11] Whitley D. An overview of evolutionary algorithms: practical issues and common pitfalls. Information and Software Technology 2001;43(14):817-831.

[12] DeJong KA. An analysis of the Behavior of a class of Genetic Adaptative System. Ph.D. thesis, University of Michigan, 1975.

[13] Morales RO, Sánchez MP, Ginori JL, Ábalo RG, Ramos R. Evaluation of QRS morphological classifiers in the presence of noise. Computers and Biomedical Research 1997; (30):200-210.

Address for correspondence:

Name: Luis David Avendaño V.

Address: Cra. 20B No. 65 A 22, Los Laureles, Manizales, Caldas, Colombia.

E-mail address: ldavendanov@unal.edu.co ldavendanov@gmail.com 